\section{Lymphocyte home movies}

Ever wanted to take a peek inside a living lymph node to see what the lymphocytes are up to? Researchers at the University of California, Irvine, have achieved just that. The groundbreaking movies, described in Science,

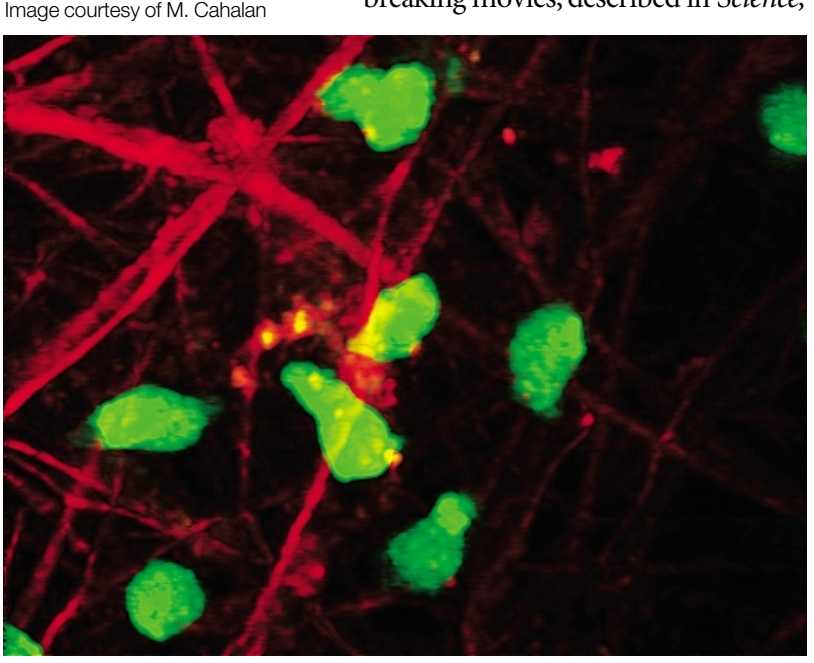

show that $\mathrm{T}$ cells dart around like schoolboy racers, whereas B cells are Sunday drivers, meandering along at a more leisurely pace.

Until now, we have been unable to visualize the activity of lymphocytes in intact lymphoid tissues, because conventional microscopes cannot penetrate more than a few $\mu \mathrm{m}$ into the tissue. Two-photon microscopy is a relatively new technology that allows high-resolution imaging of fluorescently labelled cells in tissues, at depths of up to $350 \mu \mathrm{m}$. This technique has been used previously to study neurons deep within brain tissue, and the current study is the result of a collaboration between a neurobiologist, Ian Parker, and an immunologist with a long-standing interest in lymphocyte motility, Michael Cahalan.

Purified mouse $\mathrm{T}$ and $\mathrm{B}$ cells were labelled with green (CFSE) or red (CMTMR) fluorescent dyes and injected into recipient mice. The intact lymph nodes of these mice were then isolated and analysed using three-dimensional, time-lapse, twophoton imaging.
The pattern of movement that was observed was strikingly different for $\mathrm{T}$ and $\mathrm{B}$ cells. $\mathrm{T}$ cells moved in short bursts, stopping and starting frequently, which indicates that they might pause frequently to make contacts with other cells. The average velocity of T cells was $10.8 \mu \mathrm{m} \mathrm{min}^{-1}$, and top speeds of more than $25 \mu \mathrm{m}$ $\mathrm{min}^{-1}$ were recorded. By contrast, roaming $B$ cells maintained a slower and steadier pace, having a mean velocity of $6.4 \mu \mathrm{m} \mathrm{min}{ }^{-1}$. Overall, $\mathrm{T}$ cells seemed to be more adventurous that B cells in that they explored a larger area.

These observations are of lymphocyte motion in a steady state, in the absence of antigenic challenge. To test how antigen affects T-cell motility, CFSE-labelled T-cell-receptortransgenic $\mathrm{T}$ cells were transferred into antigen-primed mice. One day after transfer, two patterns of T-cell behaviour were observed. Some enlarged $\mathrm{T}$ cells were found to be stationary within tight clusters, whereas other T cells, which were larger still, gathered in dynamic 'swarms'. The

\section{AUTOIMMUNITY}

\section{MS microarrays}

Microarray analysis of human multiple sclerosis (MS) lesions has revealed marked differences in gene expression between autopsy tissue from MS patients compared with similar tissue from individuals without MS. The authors validated two of the differentially expressed genes as potential therapeutic targets in a mouse model of MS, which established the power of the microarray approach.

MS is an autoimmune disease of the central nervous system (CNS) that results from immune-cell infiltration of the CNS white matter. Inflammation and the subsequent destruction of myelin cause the well-known symptoms of progressive paralysis. Environmental factors and genetic susceptibility are both implicated in the aberrant immune response against myelin. Histologically, two types of lesions are seen: acute lesions, which are characterized by inflammation, and chronic lesions, which show scarring and demyelination.

A comparison of messenger RNA transcripts from the two types of lesions uncovered several genes that were not thought previously to be important in MS, such as those that encode granulocyte colony-stimulating factor (G-CSF) and the $\mathrm{Fc} \gamma$ receptor. The analysis also revealed differential expression of genes in the different lesions. In all of the MS samples, the expression of thirty-nine genes was increased and the expression of 49 genes was decreased, relative to non-MS samples. Genes that had increased expression levels included those that encode the immune-response molecules G-CSF, MHC class II and immunoglobulin G; the adhesion molecules integrin- $\beta 4$ and $\mathrm{P}$-selectin; the complement components $\mathrm{Clr}$ and C3; B-cell- and macrophage-specific molecules; and several pro-inflammatory cytokines. However, comparison of the two types of lesion indicated that there is a molecular difference between acute and chronic lesions; this involves the selective upregulation of expression of 22 and 32 genes, respectively.

The authors tested the value of the microarray approach using the experimental autoimmune encephalomyelitis (EAE) mouse model of MS. Expression of the Fc $\gamma$ receptor was upregulated in chronic lesions. Fc $\gamma$ receptor-deficient EAE mice had less-severe acute disease and no chronic disease compared with wild-type mice. The expression of G-CSF was upregulated in acute lesions. The ability of G-CSF to alter the course of EAE was tested by treating the mice with G-CSF before and during the onset of disease. Early treatment decreased the severity of the disease, but later treatment had no effect, which indicates that G-CSF might be a regulatory molecule that naturally suppresses acute attacks.

Although EAE is a useful model for MS, clinical trials have shown that many efficacious therapies in mice have not translated well into humans. So, caution must be exercised in spite of the animal-model validation of two MS targets. However, this study provides proof of principle for the power of microarray technology to identify new therapeutic targets. An enormous amount of data is included in these types of reports. Databanks that contain the raw genearray data will allow more-meaningful comparisons across datasets to make the most of the increasing number of these analyses.

Melanie Brazil,Associate Editor, Nature Reviews Drug Discovery

Di) References and links ORIGINAL RESEARCH PAPER Lock, C. et al. Genemicroarray analysis of multiple sclerosis lesions yields new targets validated in autoimmune encephalitis. Nature Med. 8, 500-507 (2002) 